\title{
Size-dependent reversal of grains in perpendicular magnetic recording media measured by small-angle polarized neutron scattering
}

\author{
S. J. Lister, ${ }^{1}$ T. Thomson, ${ }^{2}$ J. Kohlbrecher ${ }^{3}$ K. Takano, ${ }^{4}$ V. Venkataramana, ${ }^{1}$ S. J. Ray, ${ }^{1}$ \\ M. P. Wismayer, ${ }^{1}$ M. A. de Vries, ${ }^{1}$ H. Do, ${ }^{4}$ Y. Ikeda, ${ }^{4}$ and S. L. Lee ${ }^{1, a)}$ \\ ${ }^{1}$ SUPA, School of Physics and Astronomy, University of St. Andrews, St. Andrews, Fife KY16 9SS, United \\ Kingdom \\ ${ }^{2}$ School of Computer Science, University of Manchester, Manchester M13 9PL, United Kingdom \\ ${ }^{3}$ Laboratory for Neutron Scattering, Paul Scherrer Institute, CH-5232 Villigen PSI, Switzerland \\ ${ }^{4}$ Hitachi San Jose Research Center, 3403 Yerba Buena Road, San Jose, California 95135, USA
}

(Received 13 July 2010; accepted 17 August 2010; published online 13 September 2010)

\begin{abstract}
Polarized small-angle neutron scattering has been used to measure the magnetic structure of a $\mathrm{CoCrPt}-\mathrm{SiO}_{\mathrm{x}}$ thin-film data storage layer, contained within a writable perpendicular recording media, at granular $(<10 \mathrm{~nm})$ length scales. The magnetic contribution to the scattering is measured as the magnetization is reversed by an external field, providing unique spatial information on the switching process. A simple model of noninteracting nanomagnetic grains provides a good description of the data and an analysis of the grain-size dependent reversal provides strong evidence for an increase in magnetic anisotropy with grain diameter. (C) 2010 American Institute of Physics. [doi:10.1063/1.3486680]
\end{abstract}

The magnetic hard disk drive (HDD) is a device of tremendous technological and commercial importance. ${ }^{1}$ Current HDD technology employs perpendicular magnetic recording where the storage media consists of a stack of sputtered thin films that are engineered to meet the stringent requirements for storing data at areal densities in the range 100-500 Gbit/in. ${ }^{2}{ }^{2}$ The recording layers are made of compositionally segregated CoCrPt-oxide thin films in which grains of a magnetic $\mathrm{CoCrPt}$ alloy are separated by a thin oxide shell, typically $\mathrm{SiO}_{2}$. The average grain diameter is typically $\langle d\rangle \sim 8 \mathrm{~nm}$ with a size distribution $\sigma /\langle d\rangle \sim 0.2$. Film thicknesses are normally in the range $11-16 \mathrm{~nm}$. These media have their magnetic moments oriented perpendicular to the plane of the film and have sufficient uniaxial perpendicular anisotropy to maintain a written bit of information against thermally activated reversal of magnetization. While recording media are widely studied by a variety of local techniques, such as Kerr microscopy ${ }^{3}$ and magnetic force microscopy (MFM) ${ }^{4}$ it is still not possible to resolve magnetic structure at length scales much below $20 \mathrm{~nm}$. We have previously shown that the use of small-angle neutron scattering (SANS) and spin-dependent SANS (SANSPoL) (Ref. 5) are very effective approaches to measure these materials. ${ }^{6,7}$ Here we use SANSPoL to probe directly the magnetic switching at a granular (sub-10 $\mathrm{nm}$ ) level.

The samples were grown by de magnetron sputtering a $16 \mathrm{~nm}$ thick, granular $\mathrm{CoCrPt}-\mathrm{SiO}_{\mathrm{x}}$ layer onto $(0.8 \mathrm{~mm}$ thick) silicon substrates. The structure included a $\mathrm{CoFe}-$ based soft magnetic underlayer onto which a thin, principally $\mathrm{Ru}$, seedlayer was deposited prior to the $\mathrm{CoCrPt}-\mathrm{SiO}_{\mathrm{x}}$ layer. The media also included a protective overcoat to inhibit oxidation. The neutron experiments were performed using the SANS-I spectrometer at the Paul Scherrer Institute (PSI), Switzerland. ${ }^{8}$ In order to provide sufficient scattering statistics, measurements were taken on a stack of 10 double-sided samples cut from the sputtered $65 \mathrm{~mm}$ disks. Neutron counts were integrated over a period of two hours for each point,

${ }^{a)}$ Electronic mail: s110@st-and.ac.uk. with data collection beginning around 1 min following a change in magnetic field. The field was aligned parallel to the neutron beam and perpendicular to the plane of the film. In this geometry the scattering vector $\mathbf{q}$ lies within the plane of the film and the $\mathbf{q}$-dependent scattering is measured along all directions within the plane using a two-dimensional multidetector. In-plane (hard axis) magnetization hysteresis loops were also measured at room temperature on an Oxford Instruments 9T vibrating sample magnetometer (VSM) with a noise floor of $1 \mu \mathrm{emu}$. The background signal due to the sample holder and substrate was subtracted by fitting a linear function to the high field region of the loop where the sample magnetization had reached saturation.

The simplest model of the magnetic switching of grains at finite temperature assumes that reversal depends solely on the size of the grain. In noninteracting systems the energy barrier to reversal is given by $\Delta E=K_{\mu} V\left[1-\left(H / H_{k}\right)\right]^{2}, 9,10$ where $K_{\mu}$ is the perpendicular anisotropy constant, $H_{k}$ $=2 K_{\mu} / \mu_{o} M_{s}$ is the effective perpendicular anisotropy field, $M_{s}$ is the saturation magnetization, and $V=\pi r_{m}^{2} t$ is the volume of the "magnetic grain" $\left(r_{m}\right.$ and $t$ being the magnetic radius and thickness of an assumed cylindrical magnetic grain). In a densely packed heterogeneous film both exchange and magnetostatic interactions must be present. However, the oxide grain boundary greatly reduces exchange interactions between grains. Additionally, the contribution from the magnetostatic (demagnetizing) field works to further reduce the effect of exchange coupling. Hence, while ignoring interaction effects clearly does not provide a complete description of media reversal, it does provide a reasonable first approximation of the switching process for the samples considered here. Within this model, a given applied field $H$ corresponds to a critical grain volume of diameter $d_{c}(H)$ below which grains will have reversed, while those of larger diameter will remain unchanged. For each field the cut-off diameter $d_{c}(H)$ in $P\left(d_{i}\right)$ may be determined by comparison of the data with the scattering function described in Ref. 7. Other patterns of switching, however, would be manifest as an alternative set of scattering curves as function of 

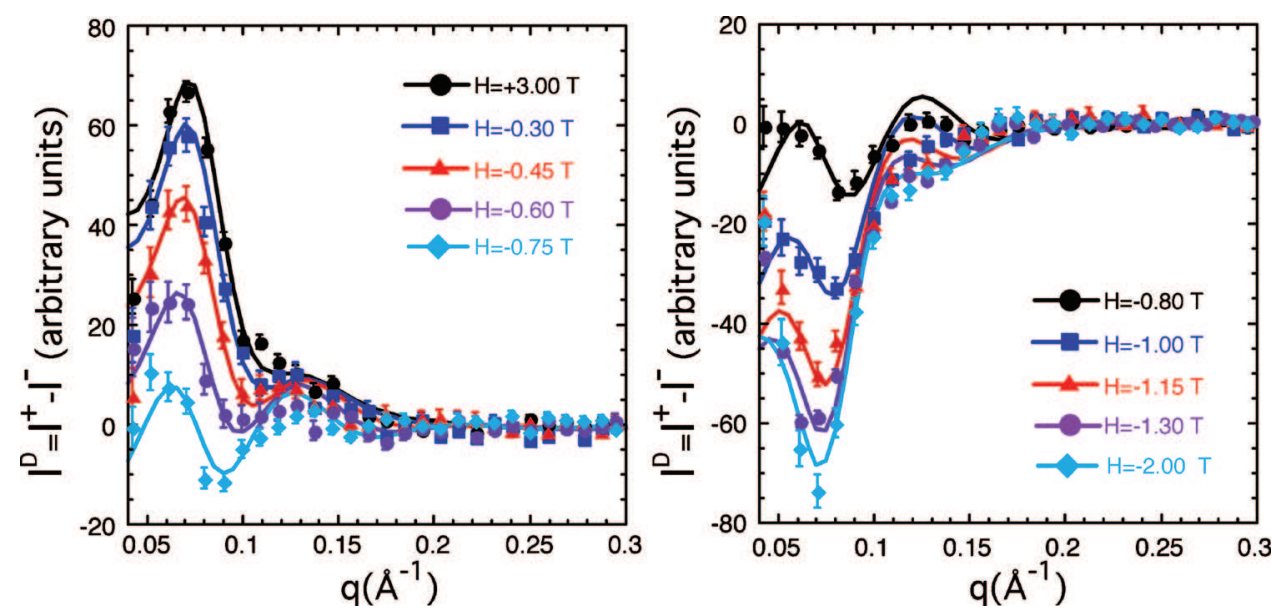

FIG. 1. (Color) The radial average of the isotropic nuclear-magnetic crossterm $I^{D}(q)$ as a function of applied magnetic field. The negative contributions to $I^{D}(q)$ that grow with increasing reverse field come from grains that have reversed their magnetization. The solid curves are derived from our scattering model in which grains of different size are permitted to switch. field. We are thus, quite generally, able to use SANSPoL to determine the size-dependent switching of the grains.

Figure 1 shows scattering data measured at a range of fields following saturation in an applied field of $H_{a p p}=+3 \mathrm{~T}$, starting with the remanent state and switching through the coercive region to a reverse field of $H_{a p p}=-2 \mathrm{~T}$. The polarized neutron scattering cross-section depends on the nuclear and magnetic form factors $f_{n}^{(i)}(q)$, $f_{m}^{(i)}(q)$ for grains of diameter $d_{i}$, which are the individual Fourier transforms of the local nuclear scattering length density $\rho_{n}\left(\mathbf{r}-\mathbf{r}_{i}\right)$ and the local magnetization $M\left(\mathbf{r}-\mathbf{r}_{i}\right)$ respectively. ${ }^{5,7}$ It is important to note that for spin-polarized neutrons the nuclear-magnetic cross-terms that we measure are sensitive to products of the form $f_{n}^{(i)}(q) f_{m}^{(i)}(q)$. $^{5,7}$ This is unlike most scattering experiments where the intensity is proportional to the square of the individual form factors. As the magnetization reverses, the sign of the magnetic contribution also reverses, hence the scattering function changes sign. This may also be understood by noting that we measure the difference $I^{D}(q)=I^{\uparrow}(q)-I^{\downarrow}(q)$ in the intensities for the two different neutron-spin polarization states $I^{\uparrow}(q), I^{\downarrow}(q)$, the relative magnitudes of which can vary with the magnetic state. ${ }^{5,7}$ Within our model one can heuristically interpret the negative part of the curves of Fig. 1 as reflecting a reversal of sign of the contributions to $I^{D}(q)$ arising from those grains that have reversed their magnetization. The basic scattering model used to fit the data, described in Ref. 7, assumes a size distribution $P\left(d_{i}\right)$ of cylindrical particles of diameter $d_{i}$ with a distribution width $\sigma /\left\langle d_{i}\right\rangle \sim 0.2$. For analytic convenience $P\left(d_{i}\right)$ is given by a Gamma-Shultz distribution, ${ }^{7,11}$ which closely resembles a log-normal distribution. This provides a good description of the field-dependent scattering curves of Fig. 1, from which we are able to determine the size dependence of the reversal process. The data are found to be consistent with a monotonically increasing cut-off diameter $d_{c}(H)$, as would be expected in the simple model discussed above.

In Fig. 2(a) we plot the experimentally determined function $d_{c}(H)$. On the same plot we include the results of using the Sharrock model (based on a Stoner Wohlfarth thermal activation model $)^{10}$ to calculate the equivalent theoretical function $d_{c}^{S W}(H, t)$ at a particular measurement time. This simply uses the assumption that for a grain of size $d_{i}$ in an applied field $H$ the rate of thermally-activated reversal is given by ${ }^{10} \quad \lambda_{i}(H)=f_{o} \exp \left[\Delta E_{i}\left(d_{i}, H\right) / k_{\mathrm{B}} T\right]$, where $\Delta E_{i}$ $=K_{\mu}^{(i)} V_{i}\left(1-H / H_{k}^{(i)}\right)^{2}$, and where we assume $H_{k}^{(i)}=H_{k}$ and hence $K_{\mu}^{(i)}=K_{\mu}$ for all grains. If this model were correct wewould expect $d_{c}(H)$ to follow the contours of constant measurement time in Fig. 2(a). As can be seen the agreement
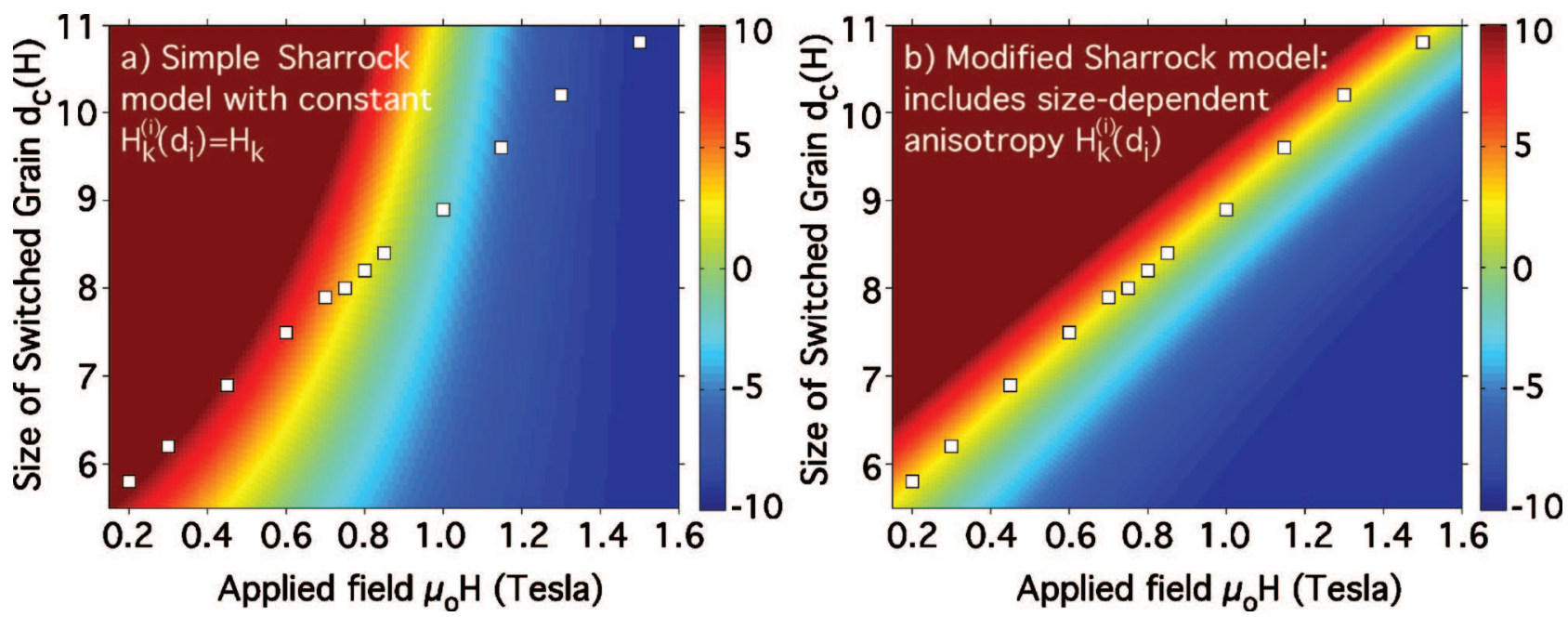

FIG. 2. (Color) The color plots of $\log _{10} t$ indicate the characteristic time $t=\ln 2 / \lambda(d, H)$ at which, following application of a reversal field $H$, a grain of diameter $d$ will have reversed its magnetization direction. For a constant measurement time values of the critical grain size $d_{c}(H)$ should thus follow the contours of constant time. (a) Measured $d_{c}(H)$ compared to a simple Sharrock model, showing poor agreement. (b) Measured $d_{c}(H)$ compared to a modified model in which the anisotropy field $H_{k}^{(i)}$ has a monotonic dependence on grain size $d_{i}$. 

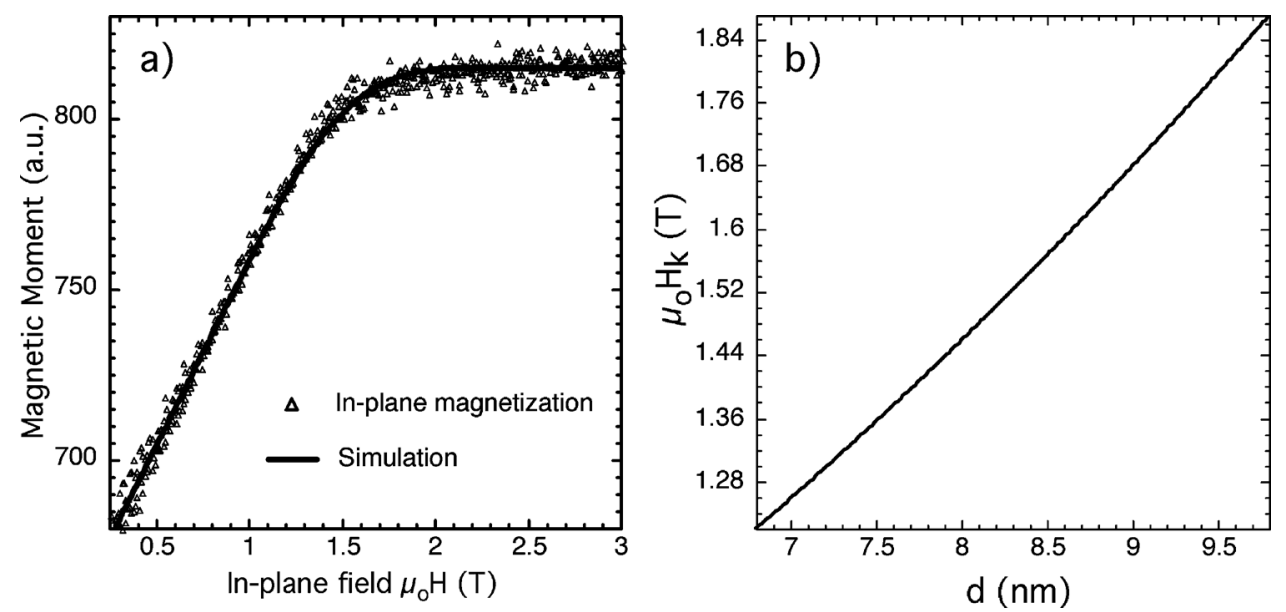

FIG. 3. (a) The in-plane magnetization curve measured using a VSM (triangles) compared to the simulation (solid line). The parameters used are those derived from neutron scattering experiments without adjustment. $M / M_{s}$ is scaled to the measured saturation moment in the curve. (b) The fitted anisotropy field $H_{k}^{(i)}$ obtained from neutron data has an approximately linear dependence on grain size $d_{i}$. with this model is rather poor. A second model where $H_{k}$ is correlated with grain diameter is shown in Fig. 2(b) where the agreement is very good. This model allows the anisotropy field $H_{k}^{(i)}$ to vary with grain size $d_{i}$. It is found that agreement can be achieved by a first order Taylor expansion of $H_{k}^{(i)}$ around $H_{k}^{(a v)}=H_{k}\left(d_{a v}\right)$ where $d_{a v}=\left\langle d_{i}\right\rangle$ is the average grain size, and where it is assumed that for all grains $M_{s}^{(i)}$ $=M_{s}$, so that $K_{\mu}^{(i)}$ scales with $H_{k}^{(i)}$. The value of $H_{k}^{(i)}$ in fact increases approximately linearly with increasing grain size $d_{i}$, which is shown in the Fig. 3(b). Since in the scattering experiments it is a cross-term that is measured, we take the value of $M_{s}$ from bulk magnetization experiments rather than from the absolute cross-section, which is $M_{s}$ $=535 \mathrm{emu} \mathrm{cm}^{-3}$ if a continuous film is assumed. However, using the additional spatial information provided by neutron measurements, this value may be further refined. Due to the $\mathrm{SiO}_{2}$ grain boundaries, the CoCrPt grains occupy only 0.8 of the volume of the layer, which leads to an average $M_{s}$ within each grain of $670 \mathrm{emu} \mathrm{cm}^{-3}$. This value of $M_{s}$, combined with the $H_{k}^{(i)}$ in our model, places the measured $d_{c}(H)$ within the correct theoretical time window for our experiment [Fig. 2(b)]. We note that the value for $M_{s}$ could be as high as $M_{s}=1040 \mathrm{emu} \mathrm{cm}^{-3}$ based on our neutron model in which the grain consists of a central region of diameter $0.8 d$ where $M_{s}$ is constant and an outer region where $M_{s}=0,{ }^{7}$ although in reality $M_{s}$ will likely be graded from the grain center to the outer rim. The calculated relaxation rates are, however, independent of this assumption.

The ability to identify a particular grain size $d_{i}$ as having switched at a particular field $H$, and hence to ascribe a particular $H_{k}^{(i)}$ to each grain, is a unique feature of the q-dependence of the neutron measurement. This information cannot be obtained via techniques that measure the total magnetic moment e.g., VSM, which must naturally integrate over all length scales. However, the reciprocal process where the $H_{k}^{(i)}$ can be used to construct magnetization curves provides a significant test of the model. In Fig. 3(a) we show the magnetization curve along the hard direction (field in the plane of the film) for the same sample. The solid line in the figure is the theoretical curve using a simple StonerWohlfarth model ${ }^{9}$ that includes the same $H_{k}^{(i)}=H_{k}^{(a v)}+\left(d_{i}\right.$ $\left.-\left\langle d_{i}\right\rangle\right) \Delta H_{k}^{(1)}+\left(d_{i}-\left\langle d_{i}\right\rangle\right)^{2} \Delta H_{k}^{(2)}$ deduced from the neutron results $\left(\mu_{o} H_{k}^{(a v)}=1.50 \mathrm{~T}, \mu_{o} \Delta H_{k}^{(1)}=0.214 \mathrm{~T} \mathrm{~nm}^{-1}, \mu_{o} \Delta H_{k}^{(2)}\right.$ $\left.=0.01 \mathrm{~T} \mathrm{~nm}^{-2},\left\langle d_{i}\right\rangle=8.2 \mathrm{~nm}\right)$ and shown in Fig. 3(b). The model has no adjustable parameters other than the moment being normalized to the measured saturated value. The agreement between the model and the data is again very good.

The agreement between such a simple (essentially Stoner-Wohlfarth) model and our data may at first seem rather surprising. However, we note that the densely packed grains in recording media are designed to be exchange decoupled, and specifically in these samples due to the presence of the oxide between the metallic alloy grains. Furthermore, the reversal mechanism at very short switching times is likely to contain elements of incoherent switching. However, at the time scale of our measurements the grains will be found in a single domain state that points either along or opposite to the field direction. By examining the state in which the grains are found even after relaxation at these very long times, it is still possible to deduce that relaxation depends on more than simply the difference in volume of the grains across the particle size distribution. In particular, we conclude that the anisotropy field of the grains $H_{k}^{(i)}$ increases with particle diameter $d_{i}$. The analysis not only provides a unique advanced characterization of these commercially important functional materials, but the results provide an excellent opportunity to inform realistic micromagnetic models of magnetic switching in future materials design.

${ }^{1}$ S. N. Piramanayagam, J. Appl. Phys. 102, 011301 (2007)

${ }^{2}$ H. J. Richter, J. Phys. D 40, R149 (2007).

${ }^{3}$ T. A. Moore, M. Kläui,L. Heyne, P. Möhrke, D. Backes, J. Rhensius, U. Rüdiger, L. J. Heyderman, T. O. Mentes, M. Á. Niño, A. Locatelli, A. Potenza, H. Marchetto, S. Cavill, and S. S. Dhesi, J. Magn. Magn. Mater. 322, 1347 (2010).

${ }^{4}$ M. Hashimoto, M. Salo, Y. Ikeda, A. Moser, R. Wood, and H. Muraoka, IEEE Trans. Magn. 43, 3315 (2007).

${ }^{5}$ A. Wiedenmann, in Neutron Scattering from Magnetic Materials, edited by T. Chatterji (Elsevier, New York, 2006), Chap. 10, p. 473.

${ }^{6}$ M. Wismayer, S. L. Lee, T. Thomson, F. Y. Ogrin, C. D. Dewhurst, S. M. Weekes, and R. Cubitt, J. Appl. Phys. 99, 08E707 (2006).

${ }^{7}$ S. J. Lister, M. P. Wismayer, V. Venkataramana, M. A. de Vries, S. J. Ray, S. L. Lee, T. Thomson, J. Kohlbrecher, H. Do, Y. Ikeda, K. Takano, and C. Dewhurst, J. Appl. Phys. 106, 063908 (2009).

${ }^{8}$ V. K. Aswal, B. van den Brandt, P. Hautle, J. Kohlbrecher, J. A. Konter, A. Michels, F. M. Piegsa, J. Stahn, S. Van Petegem, and O. Zimmer, Nucl. Instrum. Methods Phys. Res. A 586, 86 (2008).

${ }^{9}$ E. C. Stoner and E. P. Wohlfarth, Philos. Trans. R. Soc. London, Ser. A 240, 599 (1948).

${ }^{10}$ P. J. Flanders and M. P. Sharrock, J. Appl. Phys. 62, 2918 (1987).

${ }^{11}$ W. L. Griffith, R. Triolo, and A. L. Compere, Phys. Rev. A 35, 2200 (1987). 